\title{
IDENTIFICACIÓN Y FLUCTUACIÓN POBLACIONAL DEL MINADOR DE LA HOJA LIRIOMYZA TRIFOLII EN CHILE JALAPEÑO EN EL NORTE DE SINALOA
}

\author{
Fernando Alberto VAlenzuela-Escoboza1, Néstor BAUtista Martínez², \\ José Refugio LOMEli Flores², Jorge Manuel VAldez CaRrasco², \\ Edgardo Cortez MondaCa ${ }^{3}$ y Rogelio Enrique Palacios TORRES 4 \\ 1Escuela Superior de Agricultura del Valle del Fuerte, Universidad Autónoma de Sinaloa, Calle 16 \\ Ave. Japaraqui, C.P. 81110, Juan José Ríos, Ahome, Sinaloa. \\ ${ }^{2}$ Colegio de Postgraduados- Campus Montecillo Km 35.5 Carretera México - Texcoco. 56230 \\ Montecillo, Texcoco Edo. de México. \\ 3Instituto Nacional de Investigaciones Forestales, Agrícolas y Pecuarias-CEVAF- Carretera Interna- \\ cional Km 1609, CP 81110. Juan José Ríos, Guasave, Sinaloa. \\ 4Dirección de Protección Fitosanitaria- Dirección General de Sanidad Vegetal, Servicio Nacional de \\ Sanidad, Inocuidad y Calidad Agroalimentaria. Ave. Municipio Libre 377 Delegación Benito Juárez, \\ México, D.F. MÉXICO. E-mail: fervaes.69@hotmail.com
}

Valenzuela-Escoboza, F. A., N. Bautista, J. R. Lomeli Flores, J. M. Valdez, E. Cortez \& R. E. Palacios. 2010. Identificación y fluctuación poblacional del minador de la hoja Liriomyza trifolii en chile jalapeño en el norte de Sinaloa. Acta Zool. Mex. (n. s.), 26(3): 585-601.

RESUMEN. México es de los principales productores de chile, ya que al año se siembran alrededor de 140,000 ha y la producción asciende a 1'180,000 t. En el estado de Sinaloa se establecen, en promedio, 17,180 ha/año, con una producción de 488,153 t. Este cultivo es una importante fuente de empleos y de derrama económica. Se identificaron las especies de minadores de la hoja y su fluctuación poblacional en el cultivo de chile jalapeño en tres municipios del norte de Sinaloa. Se desarrollaron 29 muestreos semanales de octubre de 2008 a abril de 2009. Se contabilizaron minas en foliolos. La fluctuación de adultos se determinó con trampas de impacto color amarillo fluorescente a la altura del cultivo y una trampa fija a $1.70 \mathrm{~m}$. Se determinó taxonómicamente a Liriomyza trifolli (Burgess) mediante claves especializadas. Este agromícido presentó cantidades altas en el municipio de Guasave en comparación con Ahome y El Fuerte, aunque en los tres lotes las poblaciones más abundantes fueron en las etapas de floración y fructificación. Las trampas de altura variable capturaron más adultos del minador de la hoja en los tres lotes, en comparación con trampas fijas a $1.70 \mathrm{~m}$.

Palabras clave: Agromyzidae, Liriomyza trifolii, taxonomía, trampas de impacto, chile jalapeño.

Valenzuela-Escoboza, F. A., N. Bautista, J. R. Lomeli Flores, J. M. Valdez, E. Cortez \& R. E. Palacios. 2010. Identification and population fluctuation of the leaf miner Liriomyza trifolii in jalapeño pepper in norther Sinaloa. Acta Zool. Mex. (n. s.), 26(3): 585-601.

ABSTRACT. Mexico is one of the main producers of pepper. Near 140,000 ha are sown per year and the production rises to 1'180,000 t. In Sinaloa near 17,180 ha/per year are sown, with a production of

Recibido: 26/06/2009; aceptado: 05/08/2010. 
488,153 t. this crop is a major source of jobs and economic income. With the results of this research, we identified leaf miner species and their population fluctuation in jalapeño pepper at three locations in the north of Sinaloa. Twenty-nine weekly samplings were done from October 2008 to April 2009. Mines were counted leaflets. Adult fluctuation was determined with yellow fluorescent impact-traps at the height of the crop and another trap was set at $1.70 \mathrm{~m}$. Liriomyza trifolli (Burgues) was determined taxonomically by using specialized keys. Even though the occurrence of the agromizid miner was observed in flowers and fruits stages of jalapeño pepper plants at the three locations, it was higher in Guasave compared to Ahome and El Fuerte locations. More adult agromizids were observed on the variable high traps than on the 1.70 high ones.

Key words: Agromyzidae, Liriomyza trifolii, taxonomy, impact traps, Jalapeño pepper.

\section{INTRODUCCIÓN}

El cultivo de chile (Capsicum annuum L.) en el norte de Sinaloa es atacado por varias plagas de insectos. Recientemente los minadores de la hoja (Diptera: Agromyzidae) se han constituido como uno de los principales factores fitosanitarios que limitan la producción de este cultivo en la región, su daño llega a reducir el rendimiento y la calidad de la cosecha, sobre todo cuando se establecen desde las etapas tempranas del cultivo (Pacheco 1985). Los agromícidos son considerados insectos difíciles de ubicar taxonómicamente, debido a su pequeño tamaño y uniformidad morfológica externa, son especies estrechamente relacionadas y difíciles de separar, inclusive puede encontrarse más de una especie en una misma planta (Spencer 1973). La familia Agromyzidae cuenta con más de 2,500 especies incluidas en 28 géneros, aunque probablemente el número de especies sea mayor a 3,500 a nivel mundial (Spencer 1963). Más de 20 especies de Liriomyza han sido catalogadas como de importancia económica, y de éstas, seis son polífagas (Liu et al. 2009). En México, están registradas 30 especies de Agromyzidae y destacan por su presencia y diversidad los géneros Melanagromyza y Liriomyza (Martínez \& Étienne 2002; Palacios et al. 2008). En Sinaloa, sólo se tienen reportes de agromícidos asociados con los cultivos de tomate y canola (Alvarado \& Trumble 1999; Cortez et al. 2004), y se desconoce la identidad de las especies asociadas con el cultivo de chile, por lo que es necesario identificar y generar información al respecto.

Por lo común, las elevadas poblaciones de minador, en diversas regiones del país provocan defoliación severa de las plantas en todo el lote (Pacheco 1985; Bautista 2006). A pesar de la importancia que ha adquirido esta plaga, se carece de estudios sobre su fluctuación poblacional que ayuden a implementar técnicas de manejo oportunas y adecuadas (Barrios et al. 2004). Schuster \& Wharton (1993) observaron que en la región oeste-central de Florida, EUA, las poblaciones de minadores se incrementaron desde el inicio del desarrollo y floración en tomate fresco, lo cual atribuyeron a la aplicación de insecticidas de amplio espectro para el control de otras plagas y la consecuente eliminación de enemigos naturales. 
Es fundamental conocer la composición de especies de minador, sus técnicas de detección y fluctuación poblacional durante el cultivo, para planear medidas oportunas de manejo que controlen las plagas y logren reducir las pérdidas económicas. Los objetivos del presente trabajo fueron: identificar las especies de minadores de la hoja asociados con el cultivo de chile jalapeño en el Norte de Sinaloa, conocer su fluctuación poblacional a través del desarrollo del cultivo y determinar la mejor altura de trampas de impacto para la captura de adultos de minador.

\section{MATERIALES Y MÉTODO}

El estudio se realizó en el ciclo otoño-invierno (septiembre 2008 a abril 2009) en tres municipios del Norte de Sinaloa, México. Una parcela experimental se estableció en el Campo Experimental Valle del Fuerte del INIFAP (CEVAF), ejido Las Vacas (Juan

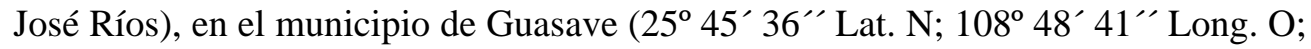
$14 \mathrm{msnm})$. La segunda parcela se estableció en el ejido Flor Azul, municipio de Ahome ( $25^{\circ} 52^{\prime} 16^{\prime \prime}$ Lat. N; $109^{\circ} 00^{\prime} 48^{\prime \prime}$ Long. O; $16 \mathrm{msnm}$ ); La tercera en una parcela escolar del Centro de Bachillerato Tecnológico Agropecuario No. 81 (CBTa 81), en el ejido " 2 de Abril", municipio El Fuerte ( $25^{\circ} 54^{\prime} 23^{\prime \prime}$ Lat. N y $108^{\circ} 56^{\prime} 13^{\prime \prime}$ Long. O; $18 \mathrm{msnm}$ ). Cada parcela experimental constó de $625 \mathrm{~m}^{2}$ sembradas la segunda quincena de septiembre de 2008 con chile jalapeño, variedad "Grande" a una densidad de $55 \mathrm{mil} \mathrm{pl} \mathrm{ha}^{-1}$. Se fertilizó previo al trasplante con $480 \mathrm{~kg} \mathrm{ha}^{-1}$ de la mezcla NPK (3:1:1); posteriormente se aplicó el fertilizante foliar Bayfolan sólido ${ }^{\circledR}$ cada 20 días. El primer riego de auxilio se realizó 10 días después del trasplante; posteriormente se realizaron riegos cada 20 días. El manejo agronómico fue el mismo que llevan a cabo los productores de la región, pero sin realizar aspersiones de insecticidas, con el propósito de que el minador de la hoja se presentara en forma natural.

Determinación de especies de minadores de la hoja en chile jalapeño. Para determinar las especies de minadores asociadas al chile jalapeño y conocer el grado de infestación del cultivo, se realizaron muestreos a intervalos semanales, de acuerdo con la técnica conocida como "cinco de oros". En cada sitio de muestreo, se seleccionaron 10 plantas pequeñas, menores de $30 \mathrm{~cm}$ y se toman cinco foliolos al azar a ambos lados de la planta, de acuerdo con la técnica propuesta por Jones \& Parrella (1986). En plantas con una altura entre 30 y $50 \mathrm{~cm}$ se subdividió cada planta en dos estratos, se tomaron cinco foliolos del estrato basal y cinco del apical; cuando el cultivo superó los $50 \mathrm{~cm}$ de altura, se realizó un muestreo estratificado en tres niveles, tomando tres folíolos del estrato basal, tres del medio y cuatro del apical. Así, se obtuvo un total de 100 folíolos por sitio y fecha de muestreo y 500 por parcela. 
Para la identificación de especies de minador de la hoja, se seleccionaron semanalmente 10 folíolos con presencia evidente de larvas de minador, en los mismos cinco sitios de cada parcela experimental (dos en cada sitio de muestreo). Se introdujeron en bolsas Ziploc ${ }^{\circledR}$ y se llevaron al laboratorio de Entomología Agrícola de la Escuela Superior de Agricultura del Valle del Fuerte (ESAVF). Las muestras se transfirieron a vasos de plástico desechables de $4.5 \mathrm{~cm}$ de altura con perforaciones en la tapa cubiertas de tela de polipropileno (Agribon ${ }^{\circledR} 17$ ), con algodón húmedo en el interior. A la emergencia de los adultos, éstos se colocaron en tubos Eppendorf de $1.5 \mathrm{~mL}$, con alcohol 70\%, se etiquetaron y se llevaron al laboratorio de Entomología Agrícola del Colegio de Postgraduados para su preparación e identificación.

La identificación de especies de Liriomyza se realiza considerando las estructuras de los genitales de los machos y la quetotaxia de la cabeza, por lo que se procedió a realizar montajes de estas estructuras, así como de tórax y abdomen de los insectos colectados. Para la preparación de los genitales se siguió la metodología propuesta por Palacios et al. (2008), los especímenes machos se maceraron en hidróxido de potasio $10 \%$ a $80^{\circ} \mathrm{C}$ durante diez min. después se lavaron y se conservaron en alcohol 70\%. Las preparaciones de edeago y bomba eyaculadora se montaron temporalmente sobre una gota de gel transparente para cabello, mezclada con una gota de glicerina. El resto de las muestras se deshidrataron gradualmente y se secaron por el método del punto crítico (Bozzola \& Russell, 1992). El estudio y fotografías se hicieron con un microscopio Tessovar y con un Fotomicroscopio III, ambos de Carl Zeiss ${ }^{\circledR}$, con una cámara digital para microscopía PaxCam3 ${ }^{\circledR}$. Otros ejemplares se observaron en un microscopio electrónico de barrido JEOL ${ }^{\circledR}$ JSM-6390, para estudiar la quetotaxia de la cabeza, el mecanismo estridulador del abdomen y los fémures posteriores.

Los especímenes adultos de minador de la hoja se identificaron mediante claves y esquemas de los genitales del macho publicados por Spencer $(1963,1973)$, Spencer \& Stegmaier (1973), Spencer \& Steyskal (1986), Spencer et al. (1992) y Palacios et al. (2008). Los resultados de las identificaciones fueron corroborados por el Dr. Rogelio Enrique Palacios Torres de la Dirección de Protección Fitosanitaria de la Dirección General de Sanidad Vegetal, Servicio Nacional de Sanidad, Inocuidad y Calidad Agroalimentaria (SENASICA). Ejemplares de resguardo del material entomológico obtenido y analizado en este estudio, se encuentran depositados en las colecciones entomológicas del Colegio de Postgraduados, Campus Montecillo, y de la ESAVF en Juan José Ríos, Ahome, Sinaloa.

Fluctuación poblacional del minador de la hoja. Para la fluctuación poblacional, el muestreo de minas, se realizó como fue descrito en el apartado anterior, para el caso de los adultos se realizaron muestreos semanales, utilizando como unidad muestral cinco trampas de impacto por parcela. Cada trampa consistió en dos 
cilindros de cartulina amarilla fluorescente de $15 \mathrm{~cm}$ de altura por $20 \mathrm{~cm}$ de perímetro, soportados en maderos de $2.5 \times 2.5 \mathrm{~cm}$ y $1.70 \mathrm{~m}$ de altura (Fig. 4). La cara externa de los cilindros se cubrió con pegamento entomológico Spider Tac ${ }^{\circledR}$ diluido con adelgazador de pinturas, el área de captura fue de $300 \mathrm{~cm}^{2}$ por trampa en el lado externo con adhesivo. Este tipo de trampas son usadas por Sanidad Vegetal en el estado de Sinaloa para el monitoreo de diversas plagas, entre ellas minadores (CESAVESIN 2008). En las trampas ubicadas verticalmente, el cilindro superior estuvo fijo a una altura de $1.70 \mathrm{~m}$, el cilindro inferior se elevó periódicamente a la altura de la copa de la planta. Las evaluaciones se hicieron semanales para estimar el número promedio de minadores por trampa por semana. Se colocaron cuatro trampas en cada esquina a $3 \mathrm{~m}$ de distancia de los bordes y una en el centro de cada lote experimental. Cada semana se retiraron y se repusieron las trampas, mismas que fueron llevadas al laboratorio de la ESAVF para contabilizar y registrar los adultos de Agromyzidae capturados. Algunos organismos se separaron de la trampa introduciendo la superficie de captura en adelgazador para pinturas. Las muestras etiquetadas se colocaron en tubos Eppendorf de $1.5 \mathrm{~mL}$. Los datos de abundancia semanal de inmaduros y adultos del minador de la hoja en cada uno de los tres lotes fue comparada mediante ANOVA (cada una de las fechas fue analizada de manera independiente). Para los datos de minas se consideró como variable dependiente el número de minas por muestra (10 hojas por cada una de las cinco repeticiones por lote) y como variable independiente la localidad. En el caso de la fluctuación de adultos, se consideró como variable dependiente el número de adultos por trampa en cada muestreo (cinco trampas por lote), y como variable independiente la localidad. Así mismo, se realizaron pruebas de " $t$ " para comparar la eficiencia de captura entre las trampas de altura fija contra las de altura variable, en este caso se consideró cada uno de los muestreos como repetición. En todos los casos se utilizó el programa Statistix 8.1 (2003) utilizando un valor de significancia de 0.05 .

\section{RESULTADOS}

Condiciones climáticas en la zona de estudio. En el municipio de Guasave, la máxima temperatura en el periodo de estudio fue de $34.3{ }^{\circ} \mathrm{C}$ y se presentó durante la fecha uno (1-oct-2008); La temperatura máxima promedio del periodo fue $28.1^{\circ} \mathrm{C}$. En la fecha 16 (14-ene-2009) se obtuvo el registro más bajo de temperatura $5.6{ }^{\circ} \mathrm{C}$, con un promedio de temperatura mínima de $12.9^{\circ} \mathrm{C}$ en el periodo de estudio. La precipitación acumulada fue de $63.2 \mathrm{~mm}$; en la fecha diez (3-dic-2008) se presentaron los máximos registros de lluvia con $23.4 \mathrm{~mm}$ y 22 días registraron lluvias apreciables en total (Fig. 1A). En el municipio de Ahome, de igual manera la máxima temperatura se presentó en la fecha uno (1-oct-2008), con $37.3{ }^{\circ} \mathrm{C}$ y el promedio de temperatura máxima en todo el periodo fue $29.1^{\circ} \mathrm{C}$. En la fecha 20 (11-feb-2009) fue registrada la más baja temperatura con $5.6{ }^{\circ} \mathrm{C}$ y una temperatura mínima promedio 
Valenzuela-Escoboza et al.: Identificación y fluctuación poblacional de Liriomyza trifolii
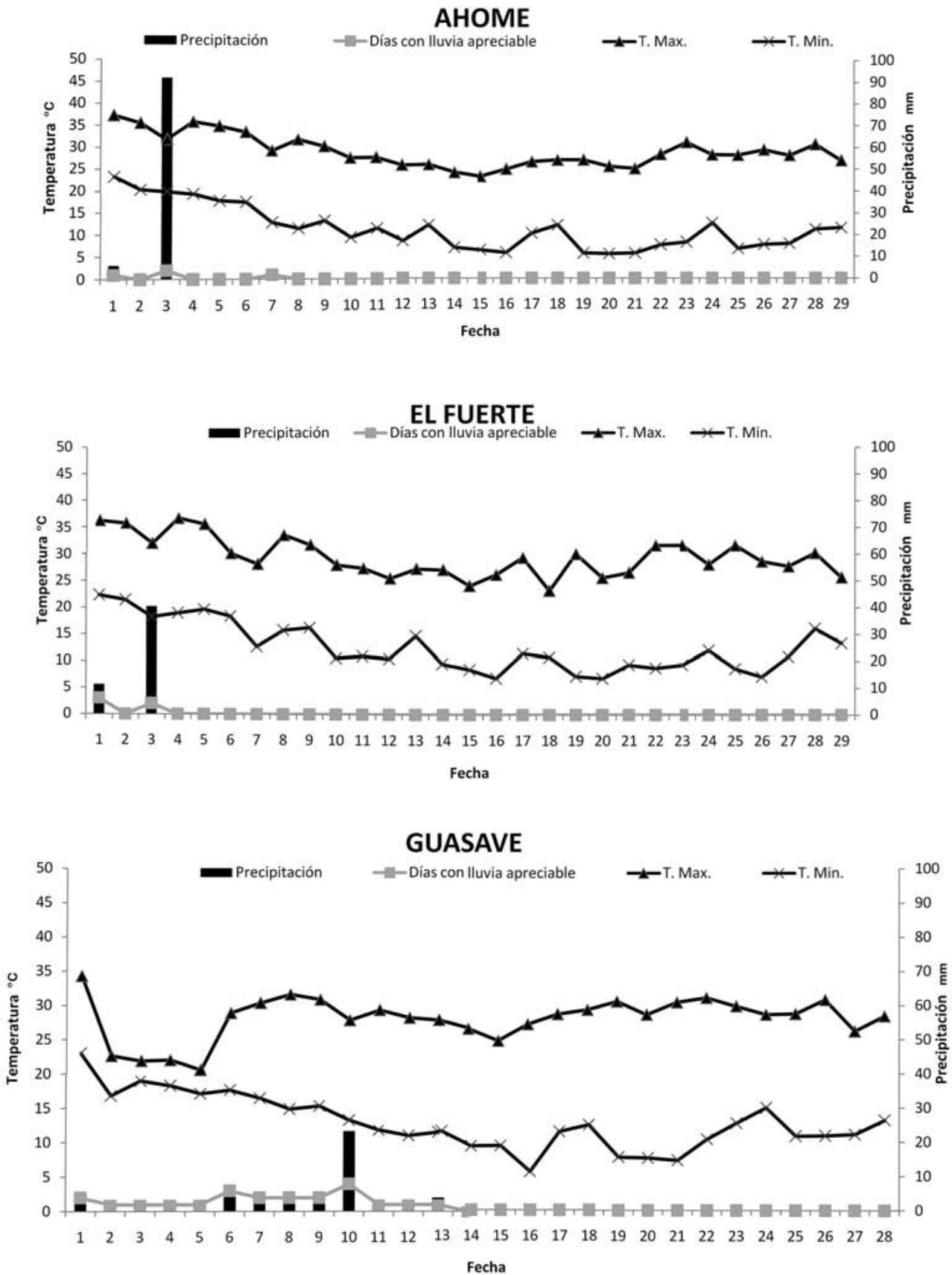

Figura 1. Datos climáticos en los municipios de Guasave, Ahome y El Fuerte. 
de $11.4{ }^{\circ} \mathrm{C}$. La precipitación acumulada en todo el periodo fue de $101.2 \mathrm{~mm}$; la máxima precipitación se registró en la fecha tres (15-oct-2008) con $91.96 \mathrm{~mm}$ en una semana, con cuatro días de lluvia apreciable en todo el periodo (Fig. 1B). En el municipio de El Fuerte, el máximo registro de temperatura se presentó en la fecha cuatro (22-oct-2008) con $36.6{ }^{\circ} \mathrm{C}$, con una temperatura máxima promedio de 29.4 ${ }^{\circ} \mathrm{C}$. La precipitación acumulada fue de $53.4 \mathrm{~mm}$; en la fecha tres (15-oct-2008) se registró la máxima precipitación con $40.4 \mathrm{~mm}$ en la semana, y en total se reportaron cinco días con lluvia apreciable (Fig. 1C).

Identificación del minador de la hoja del chile. Todos los ejemplares machos recolectados y analizados en este estudio se identificaron como Liriomyza trifolii (Burgess) (Diptera: Agromyzidae). Con la finalidad de facilitar la identificación de L. trifolii por no expertos, a continuación se presenta una diagnosis con ilustraciones que permiten la identificación inequívoca de esta especie: Cabeza. La frente y órbitas ocelares completamente amarillas, con dos pares de cerdas fronto-orbitales superiores, así como dos pares de cerdas fronto-orbitales inferiores. Las cerdas verticales emergen sobre una cutícula color amarillo. La antena presenta todos sus segmentos amarillos. En ambos sexos los ojos son glabros. El mesonoto con 3+1 pares de cerdas dorsocentrales; también se observan cuatro hileras de sedas. En las alas (Fig. 2A), la vena costa se extiende hasta la $\mathrm{M}_{1+2}$ en ambos sexos. En vista lateral, el distifalo del edeago presenta cutícula clara, con bandas esclerosadas en sus bordes superior e inferior, y en su extremo presenta una abertura amplia (Fig. 2B). El distifalo del edeago en vista ventral muestra una gran constricción en la región media y una hendidura longitudinal (Fig. 2C). La bomba eyaculadora tiene un tallo delgado que se ensancha hacia su región distal, sin rebasar el diámetro del bulbo (Fig. 2D).

\section{Fluctuación poblacional del minador de la hoja en el cultivo de chile en el Norte} de Sinaloa. De las muestras recolectadas, se obtuvieron un total de 339 adultos del minador de la hoja; $42 \%$ fueron del municipio de Guasave (85 ㅇ y 56 đ); $22 \%$ se colectaron en el municipio de Ahome (47 \& y 29 ठ $)$, y el restante $36 \%$ en el municipio de El Fuerte (63 o y 59 §). La proporción de sexos promedio fue de 1.4 hembras por cada macho; sin embargo, en el municipio de El Fuerte fue de casi un macho por cada hembra. La fluctuación poblacional de L. trifolii en los tres lotes de estudio tuvo un comportamiento similar; se presentaron las primeras infestaciones a partir de la fecha dos (8-oct-2008). Desde la fecha cinco (29-oct-2008) las poblaciones de minador de la hoja, manifestadas por minas en folíolos, tuvieron un fuerte incremento que coincidió con las etapas de floración y fructificación. Los máximos poblacionales se presentaron entre la segunda quincena de diciembre y la primera quincena de febrero, y disminuyeron a partir de la segunda quincena de febrero. Las poblaciones decrecieron drásticamente en los tres lotes a partir de 
Valenzuela-Escoboza et al.: Identificación y fluctuación poblacional de Liriomyza trifolii
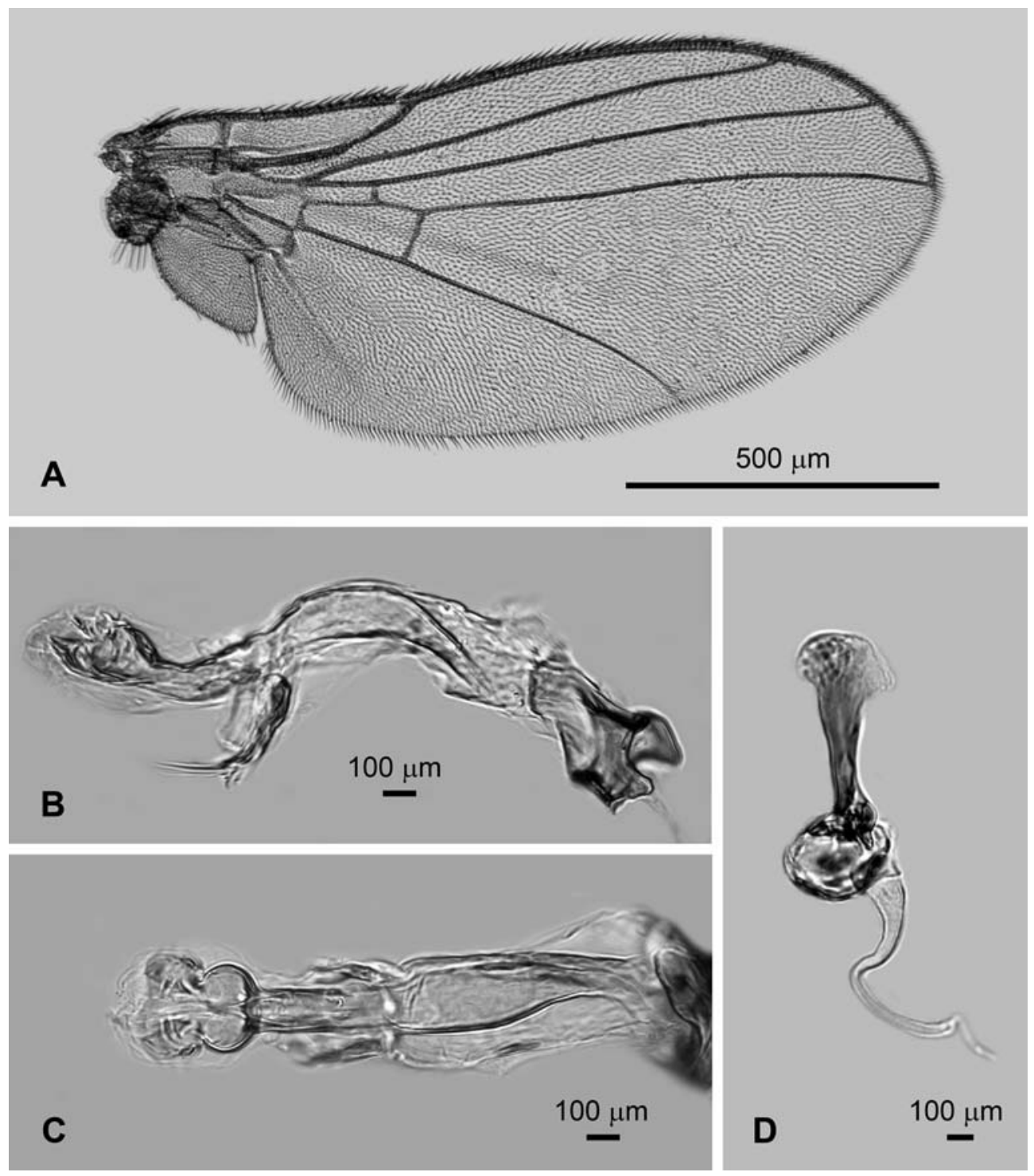

Figura 2. Liriomyza trifolii. A) Ala derecha, B) Vista lateral de edeago, C) Vista ventral de edeago, D) Vista lateral de bomba eyaculadora. 
marzo, coincidiendo con la etapa de senescencia del cultivo. En el lote de Guasave las poblaciones fueron más elevadas durante todo el estudio. Entre los meses de noviembre y diciembre, que corresponde a los periodos de crecimiento vegetativo y floración, se presentaron diferencias marcadas entre la población de Guasave y la de las otras dos localidades, que llegaron a ser de más del doble. Durante el periodo de fructificación las diferencias ya no fueron tan marcadas, pero continuaron siendo mayores las poblaciones en Guasave. La fluctuación poblacional del minador de la hoja en los lotes de Ahome y El Fuerte fueron estadísticamente similares a lo largo de los 29 muestreos realizados, pero el lote de Guasave se diferenció estadísticamente de los anteriores durante 13 muestreos, del cinco al 13 y del 25 al 29; los rangos de los valores de probabilidad obtenidos en los muestreos señalados fueron de $\mathrm{P}=0.000$ en el muestreo siete y $\mathrm{P}=0.029$ en el muestreo 13. Los muestreo 14 al 25, coincidieron con la etapa de fructificación del cultivo y los muestreos 25 al 29, con la etapa de senescencia del cultivo (Fig. 3).

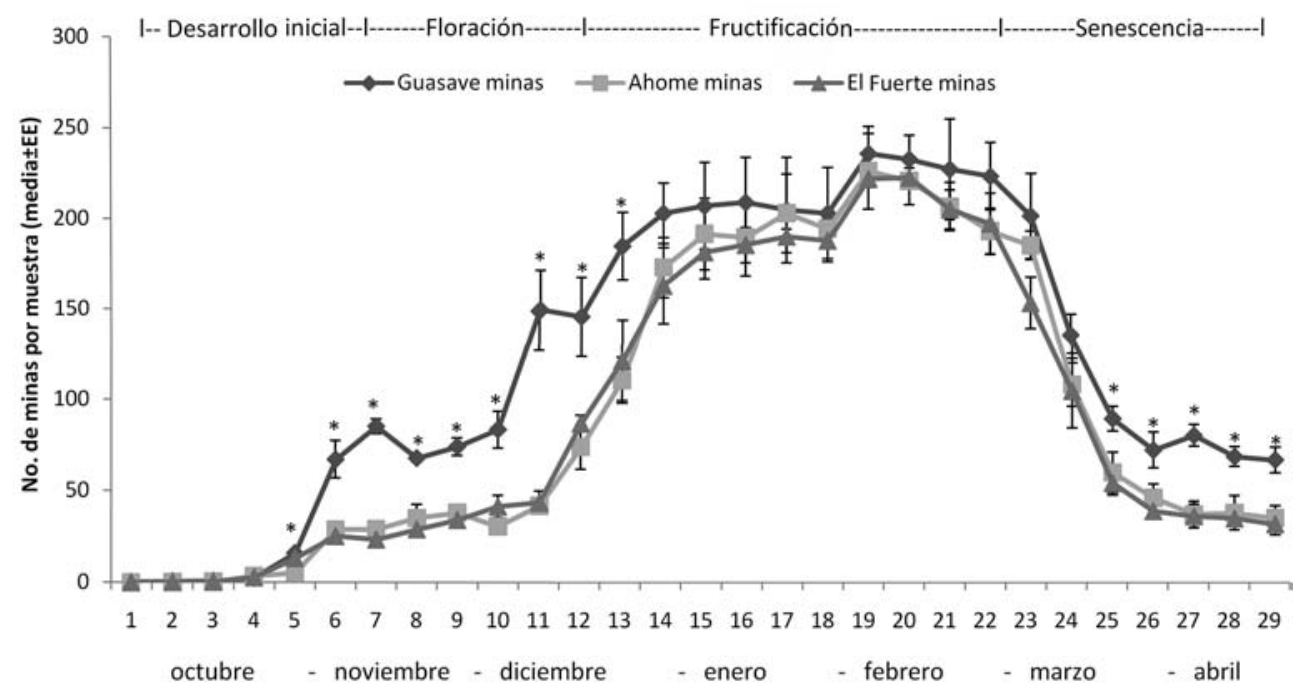

Figura 3. Fluctuación poblacional de minas del minador de la hoja en foliolos de chile jalapeño, en tres municipios del norte de Sinaloa. Los asteriscos señalan diferencias significativas entre las poblaciones de las tres localidades para cada fecha. Cada muestra consistió de 100 folíolos. 
Captura de adultos de minador de la hoja en trampas de impacto a altura fija y variable. La fluctuación de captura de adultos de minador de la hoja fue similar en los tres lotes de estudio al inicio de las evaluaciones (primeros cuatro muestreos) entre las trampas de altura fija en relación a las de altura variable, posiblemente debido a la reducida población del insecto. Sin embargo, al incrementarse las poblaciones de minadores, las diferencias fueron más amplias, existiendo en todos los casos diferencias significativas, donde los rangos de los valores de probabilidad obtenidos fluctuaron de $\mathrm{P}=0.000$ en el muestreo 14 y $\mathrm{P}=0.002$ en el muestreo 16 , entre las trampas de altura fija y las de altura variable, con mayores capturas en las trampas de altura variable, a partir del segundo mes (noviembre) y hasta el final del periodo de estudio en abril. Las trampas de altura variable fueron más eficientes en algunas fechas de muestreo hasta en más de seis veces la cantidad de especímenes que las trampas de altura fija (Fig. 4). La captura de adultos no refleja la misma fluctuación poblacional de adultos del minador en los dos tipos de trampas. En Guasave, por ejemplo, las trampas de altura variable registraron tres picos poblacionales entre los meses de diciembre y febrero, donde las máximas capturas en trampas de altura variable se presentaron en la fecha 14 (31-dic-2008) con 473 individuos en total, y promedio de 94.6 adultos/trampa/semana. En el municipio de Ahome se registraron las máximas capturas en la fecha 21 (18-feb-2009) con un total de 152 individuos, con promedio de 30.4 adultos/trampa/semana. En el municipio de El Fuerte los registros de captura muestran que la fecha 18 (28-ene-2009) fue la captura más alta con 215 individuos registrados, con promedio de 43 adultos/trampa/semana. Con relación a las trampas de altura fija, en el municipio de Guasave se presentaron los máximos registros de captura, en la fecha 14 (31-dic2008) con 92 individuos y un promedio de 18.4 adultos/trampa/semana, seguido por el municipio de El Fuerte, con máximos registros de captura en la fecha 18 (28-ene2009), con 105 individuos y un promedio de 18.6 adultos/trampa/semana. Por último, en el municipio de Ahome, se registraron las máximas capturas en la fecha nueve (26-nov-2008) con un total de 68 individuos y promedio de 13.6 adultos/trampa/semana.

\section{DISCUSIÓN}

Condiciones climáticas en la zona de estudio. En el estado de Sinaloa se presenta una temperatura media anual de $25{ }^{\circ} \mathrm{C}$ con máximas de $43{ }^{\circ} \mathrm{C}$ en los meses de agosto a septiembre y mínimas de $2{ }^{\circ} \mathrm{C}$ en enero. La precipitación media anual es de $352 \mathrm{~mm}$ y la mayor parte se registra en los meses de julio a septiembre. Comúnmente se presentan ciclones de septiembre a octubre, los cuales provocan grandes precipitaciones. El clima es cálido, seco estepario (tipo desértico) en el verano, mientras que en invierno es moderadamente frío en los meses de noviembre a enero y templado a partir de febrero (INEGI 2001; Anónimo 2003). 

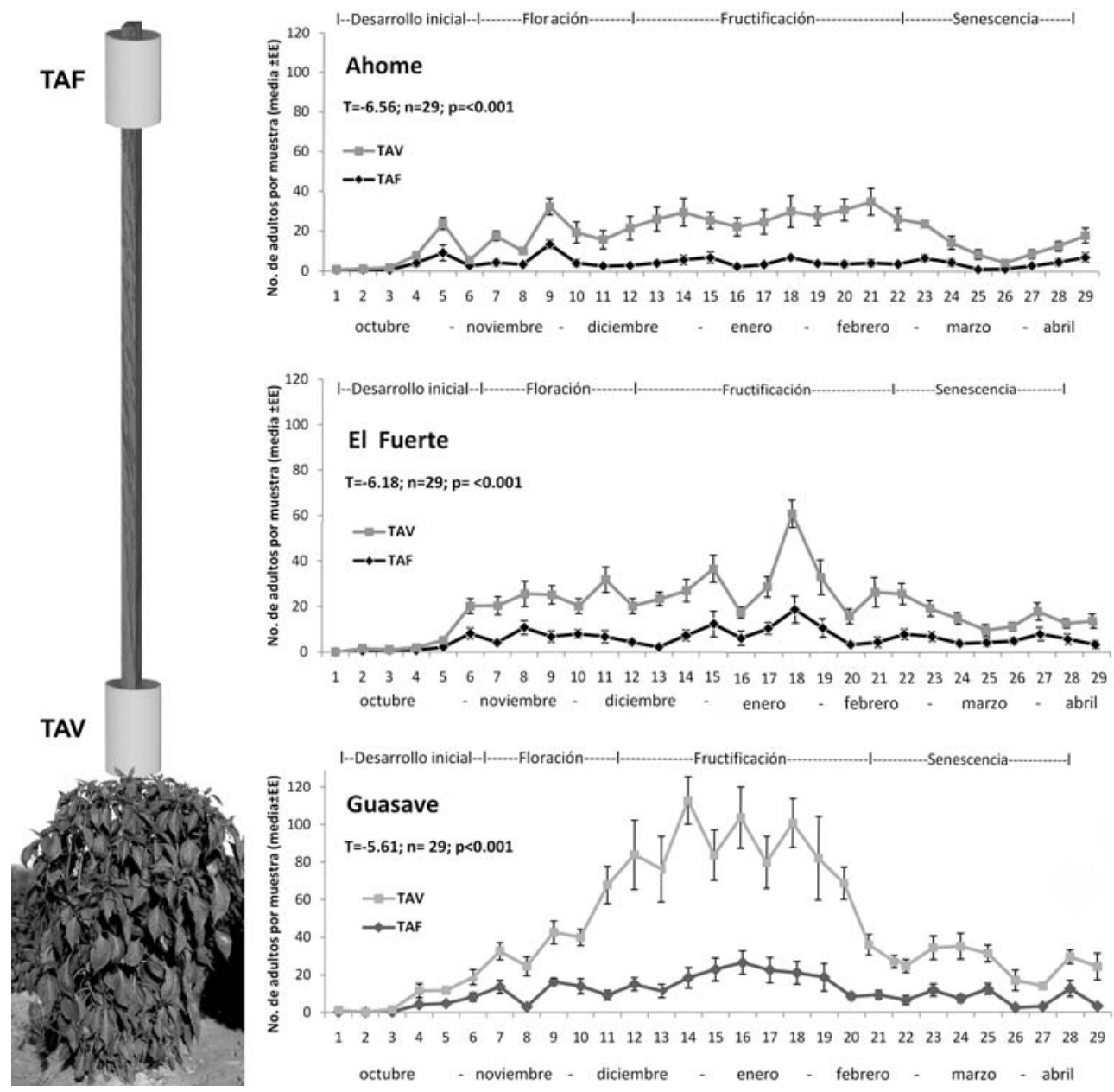

Figura 4. Comparación de la captura de adultos, del 1-oct-2008 al 15-abr-2009, en trampas de altura fija (TAF) a $1.70 \mathrm{~m}$ y trampas de altura variable (TAV), de acuerdo a la altura del cultivo, en los municipios de Guasave, Ahome y El Fuerte. Cultivo de chile jalapeño, ciclo otoño-invierno 2008-2009.

La superficie cultivada bajo sistema de riego en el estado de Sinaloa es de 831.585 ha (SAGARPA 2006). En el DDR 001 Los Mochis, se establecen 344.898 ha de cultivos como maíz blanco con 50.000 ha, frijol 22.500 ha, papa 6.675 ha, así como cultivos de chile, tomate, tomatillo, garbanzo, mango, alfalfa y otros (SAGARPA 2006). Los cultivos en el Valle del Fuerte, se establecen a partir del mes de septiembre, cumpliendo con una ventana fitosanitaria libre de cultivos hospederos 
preferidos por mosca blanca Bemisia tabaci (Genn) Biotipo "B" de junio a agosto de cada año (CESAVESIN 2008).

El cultivo de chile en la zona de estudio se establece después de la época de lluvias y durante el periodo de ejecución del presente estudio, sólo se presentaron lluvias aisladas y de poca intensidad, como sucede usualmente, pero se registraron diferencias importantes en la distribución e intensidad de las mismas. En el municipio de Ahome se presentó la mayor precipitación acumulada, $101.2 \mathrm{~mm}$; mientras que en El Fuerte y Guasave fue menor a los $64 \mathrm{~mm}$. Con relación al número de días con lluvia apreciable (es decir $>0.5 \mathrm{~mm}$ ), en el municipio de Guasave se presentaron 22 días mientras que en Ahome y El Fuerte sólo fueron cuatro y cinco, respectivamente. Tanto en Ahome como en El Fuerte la precipitación se presentó al inicio del cultivo con un máximo de $45 \mathrm{~mm}$ en una semana para el primero y de 40 para el segundo; lo anterior contrasta con lo sucedido en Guasave, ya que las primeras lluvias apreciables se presentaron hasta el segundo mes y nunca fueron superiores a los $30 \mathrm{~mm}$ por semana, sin embargo, fueron continuas durante cinco semanas, de las fechas de muestreo seis a la diez (Fig. 1). Por otro lado, las lluvias continuas entre la semana seis a la diez, pudieron reducir algún factor de mortalidad del minador, e incluso el mayor número de precipitaciones a lo largo del desarrollo del cultivo. Probablemente la ausencia de lluvias en el primer mes pudo afectar el establecimiento temprano de las poblaciones de minador en esta última localidad. El efecto de diversos factores climatológicos fue revisado por Lomeli et al. (2009) quienes hacen un análisis de los factores de mortalidad de los minadores de la hoja, encontraron que los factores abióticos como heladas, humedad y lluvias fuertes tienen efecto negativo en las poblaciones plaga y su comportamiento de apareamiento y oviposición, provocando disminuciones de población drásticas aunque por periodos cortos. Con relación a la temperatura, las máximas se presentaron durante los primeros días del cultivo en todas las regiones estudiadas. Tanto en los municipios de Ahome y El Fuerte las máximas se mantuvieron prácticamente estables entre 30 y $35^{\circ} \mathrm{C}$, mientras que las mínimas oscilaron entre 20 y $25^{\circ} \mathrm{C}$. En contraste, Guasave presentó temperaturas más fluctuantes. Durante el primer mes se presentó un periodo de temperaturas máximas cercanas a $25^{\circ} \mathrm{C}$ y las mínimas cercanas a $20^{\circ} \mathrm{C}$. Esta condición pudo haber favorecido el establecimiento de las poblaciones de minador en esta región, Milla \& Reitz (2005) indican que el umbral mínimo de desarrollo para especies de Liriomyza es $8.1{ }^{\circ} \mathrm{C}$ y su umbral máximo es $29.5^{\circ} \mathrm{C}$, con una temperatura óptima de desarrollo de $25^{\circ} \mathrm{C}$.

Identificación del minador de la hoja del chile. L. trifolii fue la única especie encontrada en los muestreos de campo en los cultivos de chile. Junto con $L$. huidobrensis y L. sativae, están consideradas como cosmopolitas y han sido localizadas en EUA, Irán, Argentina y Japón como unas de las principales plagas en 
cultivos hortícolas y florícolas (Chandler \& Gilstrap 1987; Reitz \& Trumble 2002; Schuster \& Wharton 1993; Asadi et al. 2006; Abe \& Konishi 2004; Kashiwagi et al. 2005; Salvo \& Valladares 2007). En México L. trifolii es una especie común y ha sido notificada por varios autores como plaga de diversos cultivos en Sinaloa y el Noroeste de México (Pacheco 1985; Alvarado \& Trumble 1999, Cortez et al. 2004). En un estudio reciente, esta misma especie fue encontrada como plaga del cultivo de chile, donde son necesarias aplicaciones de productos químicos para su control, además se menciona al Sur del estado de Tamaulipas como su distribución geográfica más precisa (Palacios et al. 2008).

Fluctuación poblacional del minador de la hoja en el cultivo de chile. Las poblaciones del minador fueron extremadamente bajas al inicio del cultivo $(<$ de 2 minas), por lo que se considera que la colonización del cultivo del chile se dio después de casi un mes de su establecimiento. En Sinaloa existe una ventana fitosanitaria libre de cultivos hospederos preferidos por mosca blanca implementada por primera vez el año de 1995 y posteriormente impuesta de nuevo el año 2006, misma que sigue vigente, con el fin de evitar la proliferación de mosquita blanca y otras plagas (Cesavesin 2008). Esta medida fitosanitaria procura que en la región no se siembre algún cultivo de hoja ancha por un espacio de tres meses durante el año (junio a agosto). Esta condición posiblemente evitó que los minadores pudieran infestar los cultivos de manera inmediata después del trasplante del chile. Por otra parte en los municipios de Ahome y El Fuerte se presentaron lluvias durante el primer mes; esta condición pudo ser la causa de que las poblaciones del minador no crecieran tan rápido como en Guasave. Así mismo, los primeros meses se presentaron en Guasave condiciones de temperaturas menores de $30{ }^{\circ} \mathrm{C}$, lo cual también pudo contribuir a que en este municipio la colonización se realizara en menor tiempo y de manera más agresiva. La precipitación también puede explicar las disminuciones de las poblaciones del minador en Guasave en las fechas ocho a 11 (Fig. 1). Trabajos previos han explorado el efecto de factores climáticos sobre minadores; Parrella (1984) evaluó el efecto de temperaturas constantes (15.6, 21.1, 26.7, 32.2 y $\left.37.8{ }^{\circ} \mathrm{C}\right)$ sobre la oviposición, alimentación y longevidad de L. trifolii en crisantemo; encontró que la oviposición fue similar a 21.1, 26.7 y $32.2{ }^{\circ} \mathrm{C}$ pero fue significativamente reducida a 15.6 y $37.8{ }^{\circ} \mathrm{C}$, lo que confirma el efecto de la temperatura en la oviposición de la plaga. Además, otros autores señalan que la tasa de supervivencia de los insectos se reduce con la lluvia (Lomeli et al. 2009).

Durante la etapa de fructificación se presentaron las poblaciones más altas, esto puede deberse a la mayor disposición de hojas, alimento y refugio para el minador de la hoja. El presente estudio muestra la misma tendencia que lo observado en la región de Weslaco, Texas, EUA, donde L. trifolii tuvo sus primeras infestaciones en la etapa de crecimiento inicial y su presencia y daño se fueron incrementando 
conforme las plantas aumentaban de tamaño; por lo tanto, ellos observaron las máximas poblaciones de larvas y minas en las etapas de desarrollo vegetal y reproductivas del cultivo de chile Bell (Chandler \& Gilstrap 1987). Para 1974 en Florida, EUA, se evaluó semanalmente la fluctuación poblacional de adultos y minas de Liriomyza sp, en tomate, berenjena, papa y chile, así como leguminosas, cucurbitáceas y brasicáceas, de la misma manera que aquí, el mayor porcentaje de adultos se presentó en las etapas de desarrollo y reproductivas de los cultivos, y decreció en la etapa de senescencia de los mismos (Musgrave et al. 1975).

Comparación de adultos capturados en trampas fijas y móviles. Las trampas amarillas permitieron definir que la mayor captura de adultos a lo largo del ciclo del cultivo (Fig.4), ocurrió en las trampas de altura variable, más cercanas al dosel de las plantas; lo anterior parece lógico pues los adultos del minador de la hoja se deben concentrar cercanos al dosel, donde buscan el lugar para alimentarse y para ovipositar, lo que garantiza el desarrollo de su progenie. En Costa Rica, Gómez y Rodríguez (1995) evaluaron la efectividad de las trampas cromáticas amarillas colocadas en tres estratos del cultivo de papa para el monitoreo de minadores; los resultados coinciden con los de este estudio, ya que las máximas capturas se obtuvieron en trampas colocadas al nivel del dosel del cultivo. Así mismo, en la etapa de prefloración y floración se registraron las máximas poblaciones de la plaga. El empleo de trampas de impacto amarillas con pegamento entomológico se utilizó por primera vez para detectar adultos de minador del género Liriomyza por Musgrave y colaboradores (1975). Estudios subsecuentes han confirmado que el color amarillo en trampas pegajosas es el más efectivo en la captura de minadores (Larraín \& Muñoz 1997; Risco et al. 1999; Martin et al. 2005). Los resultados de este trabajo, demuestran que la captura de adultos de minador de la hoja fue similar en los tres lotes de estudio al inicio de las observaciones. Las capturas se incrementaron en todos los lotes a partir de noviembre de 2008; pero el número de minadores capturados fue significativamente mayor durante todo el periodo de investigación. Las capturas más abundantes de adultos de minador de la hoja tanto en trampas de altura variable como en trampas de altura fija, en los tres lotes experimentales, coincidieron con las etapas de floración y fructificación del cultivo de chile cuando las plantas presentan mayor cantidad de hojas, lo que se traduce en mayor cantidad alimento y refugio para el insecto plaga. Por otra parte, Larraín y Muñoz (1997), encontraron una correlación entre las máximas capturas de adultos de minador en trampas de pegamento amarillas y la mayor cantidad de daño de larvas, como galerías y oxidación del tejido foliar; al igual que en el presente estudio, sus poblaciones declinaron a la senescencia del cultivo.

A manera de conclusiones, $L$. trifolii es la especie de minador de la hoja asociada al cultivo de chile jalapeño en los tres municipios del Norte de Sinaloa. La 
fluctuación poblacional en minas por foliolos y adultos capturados en trampas de impacto, mostró un incremento paulatino a partir de noviembre de 2008, llegando a sus máximos poblacionales en enero y febrero, coincidiendo con las etapas de floración y fructificación del cultivo, decreciendo posteriormente en la etapa de senescencia del mismo. La abundancia de minador de la hoja fue significativamente mayor en el lote establecido en el municipio de Guasave, probablemente debido a condiciones de clima. Las trampas de altura variable de acuerdo a la altura del cultivo, fueron mas eficientes para capturar adultos de minador de la hoja que las trampas de altura fija $(1.70 \mathrm{~m})$.

AGRADECIMIENTOS. Se agradece al Consejo Nacional de Ciencia y Tecnología (CONACYT) por el apoyo brindado para la realización del presente estudio (número de registro 252407), INIFAP- Campo Experimental Valle del Fuerte, a las autoridades del CBTa 81 y al productor agrícola Ismael Talamantes Apodaca por facilitar los lotes para realizar el presente estudio.

\section{LITERATURA CITADA}

Abe, Y. \& K. Konishi. 2004. Taxonomic notes on Gronotoma (Hymenoptera: Eucoilidae) parasitic on the serpentine leafminer, Liriomyza trifolii (Diptera: Agromyzidae). ESAKIA, (44): 103-110.

Alvarado, R. B. \& J. T. Trumble. 1999. El manejo integrado de las plagas: Un ejemplo en el cultivo de tomate en Sinaloa. Pp. 435-449. In: S. Anaya, R. y R. Nápoles (Eds). Hortalizas, plagas y enfermedades. Edit. Trillas. ISBN 968-24-1283-8. México, D.F.

Analytical Software. 2003. Statistix 8.1 User's Manual. Tallase, Florida USA.

Anónimo. 2003. Fundación produce. Guía para la asistencia técnica agrícola para el área de influencia del campo experimental valle del Fuerte. Sexta Edición. Sinaloa, México. 208 p.

Asadi, R., A. A. Talebi., Y. Fathipour, S. Moharramipour \& E. Rakhshani. 2006. Identification of the Agromyzid Leafminers Genus Liriomyza (Dip.: Agromyzidae) in Varamin, Iran. Journal of Agricultural, Science and Technology, 8: 293-303.

Barrios, D. B., R. Alatorre R., H. Calyecac. C., \& N. Bautista M. 2004. Identificación y fluctuación poblacional de plagas de col (Brassica aleracea var. Capitata) y sus enemigos naturales en Acatzingo, Puebla. Agrociencia, 38(2): 239-248.

Bautista, M. N. 2006. Insectos plaga, Una guía ilustrada para su identificación. Colegio de Postgraduados. ISBN: 968-839-489-0. Montecillo, Texcoco. Edo. de México. 113 p.

Bozzola, J.J. \& L. D. Russell. 1992. Electron microscopy. Jones and Bartlett Publishers. 542 pp.

Chandler, L. D. \& F. E. Gilstrap. 1987. Seasonal fluctuations and age structure of Liriomyza trifolii (Diptera: Agromyzidae) larval populations on bell peppers. Journal of Economic Entomology, 80: 102-106.

Comité Estatal de Sanidad Vegetal del Estado de Sinaloa (CESAVESIN). 2008. Ventana fitosanitaria de junio a agosto. Culiacán, Sinaloa, 1(2): 22 pp.

Cortez, M. E., N. Castillo T. \& J. Macias C. 2004. Enemigos naturales para el control biológico de insectos plaga en canola. INIFAP-CIRNO-CEVAF. Folleto Técnico No 22: 18-22.

Gómez, Y. \& C. Rodríguez. 1995. Actividad de Liriomyza huidobrensis de acuerdo a las diversas horas del día y tipos de muestreo en relación a la fenología del cultivo de papa (Solanum tuberosum). Agronomía Mesoamericana, 6: 32-49.

INEGI. 2001. Anuario estadístico Sinaloa. Gobierno del Estado de Sinaloa. 524 p. 
Jones, V. P. \& M. P. Parrela. 1986. Development of sampling strategies for larvae of Liriomyza trifolii (Diptera: Agromyzidae) in chrysanthemus. Environmental Entomology, 15: 268-273.

Kashiwagi, T., Y. Horibata., D. B. Mekuria., S. Tebayashi \& C. Kim. 2005. Ovipositional deterrent in the sweet pepper, Capsicum annuиm, at the mature stage against Liriomyza trifolii (Burgess). Bioscience, Biotechnology and Biochemistry, 69(10): 1831-1835.

Larraín, S. P. \& C. Muñoz. M. 1997. Abundancia estacional, hospederos alternativos y parasitismo de Liriomyza huidobrensis (Blanchard) (Diptera: Agromyzidae), en los cultivos de papa de la IV región de Chile. Agricultura Técnica (Chile), 57(4): 290-296.

Liu, T. X., L. Kang., K. M. Heinz \& J. Trumble. (2009). Biological control of Liriomyza leafminers: progress and perspective. Perspectives in Agriculture, Veterinary Science, Nutrition and Natural Resources, 4 (4): 1-16.

Lomeli, F. J. R., J. F. Barrera \& J. S. Bernal. 2009. Impact of natural enemies on coffee leafminer Leucoptera coffeella (Lepidoptera: Lyonetiidae) population dynamics in Chiapas, México. Biological Control, 51: 51-60.

Martin, A. D., R. S. Vernon \& R. H. Hallett. 2005. Influence of colour and trap height on captures of adult pea leafminer, Liriomyza huidobrensis (Blanchard) (Diptera: Agromyzidae), in celery. Journal of the Entomological Society of Ontario, 136: 25-35.

Martínez, M. \& J. Étienne. 2002. Liste systématique et biogéographique des Agromyzidae (Diptera) de la région néotropicale. Bollettino di Zoologia Agraria e di Bachiccoltura, Serie II, 34(1): 25-52.

Milla, K. \& S. Reitz. 2005. Spatial/temporal model for survivability of pea leafminer (Liriomyza huidobrensis) in warm climates: a case study in south Florida, USA. European Journal of Scientific Research, 7(5): 65-73.

Musgrave, C. A., S. L. Poe \& D. R. Bennett. 1975. Leaf miner population estimation in polycultured vegetables. Florida State Horticultural Society, 7076: 56-60.

Pacheco, M. F. 1985. Plagas de los cultivos agrícolas en Sonora y Baja California. SARH-INIFAPCIANO-CAEVY. Libro Técnico No. 1. Cd. Obregón, Sonora, Méx. 414 p.

Palacios, T. R. E., J. Romero N., J. Étienne., J.L. Carrillo S., J. M. Valdez C., H. Bravo M., S. D. Koch., V. López M. \& A. P. Terán V. 2008. Identificación, distribución y plantas hospederas de diez especies de agromyzidae (Insecta: Diptera), de interés agronómico en México. Acta Zoológica Mexicana (n. s.), 24 (3): 7-32.

Parrella, M. P. 1984. Effect of temperature on oviposition, feeding, and longevity of Liriomyza trifolii (Diptera: Agromyzidae). Canadian Entomologist, 116: 85-92.

Reitz, S. R. \& J. T. Trumble. 2002. Interspecific and intraspecific differences in two Liriomyza leafminer species in California. Entomología Experimentalis et Applicata,102(2):101-113.

Risco, B., E. Sierra., A. M. Golmirzale., J. Tenorio., M. Palacios \& K. V. Raman. 1999. Control de Liriomyza huidobrensis Blanchard (Diptera: Agromyzidae) con trampas pegajosas fijas y móviles en el cultivo de papa en Ica, Perú. Revista Peruana de Entomología, 1999.

SAGARPA, 2006. Secretaría de Agricultura, Ganadería y Pesca del Gobierno del Estado -OEIDRUSen línea .Disponible en: http://www.oeidrus-sinaloa.gob.mx/ ( consultado 20-julio-2008).

Salvo, A. \& G. R. Valladares. 2007. Parasitoides de minadores de hojas y manejo de plagas. Ciencia e Investigación Agraria, 34(3): 167-185.

Schuster, D. J. \& R. A. Wharton. 1993. Hymenopterous parasitoids of leaf-mining liriomyza spp. (Diptera: Agromyzidae) on tomato in Florida. Environmental Entomology, 22(5): 1188-1191.

Spencer, K. A. 1963. A synopsis of the neotropical Agromyzidae (Diptera). Transactions of the Royal Entomological Society of London, 115(12): 291-389.

Spencer, K. A. 1973. The Agromyzidae (Diptera) of Venezuela. Revista de la Facultad de Agronomía (Maracay), 7(2): 5-108. 
Spencer, K. A. \& C. E. Stegmaier. 1973. Agromyzidae of Florida, with a supplement on species from the Caribbean. Arthropods of Florida and neighboring land areas. Florida Department of Agriculture and Consumer Services. Gainesville, Florida, U.S.A. 205 pp.

Spencer, K. A. \& J. C. Steyskal. 1986. Manual of the Agromyzidae (Diptera) of the United States. United States Department of Agriculture. Washington. D. C. Agriculture Handbook. 638 pp.

Spencer, K. A., M. Martínez \& J. Étienne.1992. Les Agromyzidae (Diptera) de Guadeloupe. Annales de la Société Entomologique de France (N.S.), 28(3): 251-302. 